\title{
EDUCAÇÃO INTERPROFISSIONAL EM SAÚDE NO ENSINO SUPERIOR: REVISÃO INTEGRATIVA SOBRE A EXPERIÊNCIA BRASILEIRA
}

\author{
INTERPROFESSIONAL EDUCATION IN HEALTH IN HIGHER \\ EDUCATION: INTEGRATIVE REVIEW ON BRAZILIAN \\ EXPERIENCE
}

\section{EDUCACIÓN INTERPROFESIONAL EN SALUD EN LA EDUCACIÓN SUPERIOR: REVISIÓN INTEGRATIVA DE LA EXPERIENCIA BRASILENA}

Xavéle Braatz Petermann ${ }^{1}$

https://orcid.org/0000-0003-2672-0164

Silvana Basso Miolo ${ }^{2}$

https://orcid.org/0000-0003-2864-7490

1 Faculdade Dom Alberto, Santa Cruz do Sul, Rio Grande do Sul - Brasil. E-mail: xavelepetermann@gmail.com.

2 Prefeitura Municipal de Santa Maria, Santa Maria, Rio Grande do Sul - Brasil. E-mail: sbmiolo@ hotmail.com.

\section{Resumo}

O objetivo deste estudo foi analisar a produção científica sobre educação interprofissional em saúde no Brasil no contexto do ensino superior. Para isso realizou-se um estudo de revisão integrativa da literatura. Foram considerados estudos nacionais, com texto em línguas portuguesa, espanhola e inglesa, publicados de 2004 a 2020 nas bases de dados SciELO e LILACS, sendo incluídos nesse estudo de revisão integrativa 16 artigos. Os resultados foram avaliados pela técnica da análise temática. As categorias que denotaram a produção científica sobre educação interprofissional no ensino superior no Brasil compreenderam a educação interprofissional como meio para a troca de saberes, como espaço de reflexão e como elemento para o cuidado integral. Os resultados encontrados retrataram a importância da educação interprofissional no contexto analisado, com impacto positivo sobre o processo de ensino-aprendizagem na educação superior em saúde e, consequentemente, sobre a qualidade da atenção prestada na assistência à saúde. 
Palavras-chave: Educação Interprofissional. Aprendizado Colaborativo. Educação Superior. Saúde. Revisão.

\begin{abstract}
The aim of this study was to analyze the scientific production on Interprofessional Education in Health in Brazil in the context of higher education. For this end, an Integrative Literature Review study was carried out. National studies were considered, with text in Portuguese, Spanish and English, published from 2004 to 2020 in the SciELO and LILACS databases, and 16 articles were included in this integrative review study. The results were analyzed using the thematic analysis technique. The categories that denote the scientific production on Interprofessional Education in higher education in Brazil understood Interprofessional Education as a means for the exchange of knowledge, as a space for reflection and as an element for comprehensive care. The results found portrayed the importance of Interprofessional Education in the analyzed context, with a positive impact on the teaching-learning process in higher education in health and, consequently, on the quality of care provided in health care.
\end{abstract}

Keywords: Interprofessional Education. Interdisciplinary Placement. Higher Education. Health. Review.

\title{
Resumen
}

El objetivo de este estudio ha sido analizar la producción científica sobre educación interprofesional en salud en Brasil en el contexto de la educación superior. Para ello, se realizó un estudio de revisión integradora de literatura. Se consideraron estudios nacionales, con textos en portugués, español e inglés, publicados de 2004 hasta 2020 en las bases de datos SciELO y LILACS, y se incluyeron 16 artículos en este estudio de revisión integradora. Los resultados se analizaron mediante la técnica de análisis temático. Las categorías que denotan la producción científica sobre educación interprofesional en la educación superior en Brasil entendieron la educación interprofesional como un medio para el intercambio de conocimientos, como un espacio de reflexión y como un elemento de atención integral. Los resultados encontrados retrataron la importancia de la educación interprofesional en el contexto analizado, con un impacto positivo en el proceso de enseñanza-aprendizaje en la educación superior en salud y, en consecuencia, en la calidad de la atención brindada en el cuidado de la salud.

Palabras clave: Educación Interprofesional. Aprendizaje Colaborativo. Educación Universitaria. Salud. Revisión.

\section{Introdução}

A educação interprofissional (EIP) surgiu como uma nova proposta que se distingue da educação profissional tradicional e se caracteriza pela produção do conhecimento a partir da integração entre diferentes categorias profissionais no contexto do ensino-aprendizado 
(CAMARA; GROSSEMAN; PINHO, 2015). Olson et al. (2014) a definem como uma “ocasião em que duas ou mais profissões aprendem com, para e sobre a outra para melhorar a colaboração e a qualidade dos cuidados".

Reeves (2016) relata que a EIP oferece oportunidades únicas aos discentes para o aprendizado em conjunto com outros profissionais com o objetivo de promover atitudes e habilidades para o trabalho em equipe. Ainda ressalta que o trabalho coletivo é uma alternativa eficaz para a garantia de um cuidado de alta qualidade e com segurança para a população, em especial, no contexto do Sistema Único de Saúde (SUS) (REEVES, 2016).

Em nível internacional, o debate sobre a EIP é recente. Alguns teóricos atribuem um movimento inicial a um grupo de estudiosos da Organização Mundial da Saúde (OMS) em meados da década de 1980. Outros autores descrevem que os estudos se iniciaram na década de 1960, no Reino Unido, sendo a EIP associada ao imperativo de se encontrarem estratégias para a superação da fragmentação do trabalho em saúde (REEVES, 2016).

No Brasil, a incorporação da EIP ainda ocorre de maneira tímida (PEDUZZI, 2016). Em 2004, foi instituída a Política Nacional de Educação Permanente em Saúde (PNEPS), caracterizando-se como uma importante política pública de reorientação das práticas e da formação em saúde e criando um ambiente propício para a incorporação e o fortalecimento da EIP. Foi apenas em 2016, no entanto, que a Organização Pan-americana de Saúde (OPAS) convocou seus estados-membros a conhecerem, debaterem e implementarem a EIP em seus territórios (PAHO, 2017).

Sendo assim, a partir de 2017, motivado pela OPAS, o Ministério da Saúde definiu um plano de ação brasileiro para a inclusão da EIP no processo de formação em saúde, tendo como linhas de ação: fortalecimento da EIP como meio para a reorientação profissional; levantamento das iniciativas de EIP no Brasil; desenvolvimento docente para EIP; fortalecimento dos espaços de divulgação e produção do conhecimento em EIP; e EIP nos espaços de educação permanente em saúde (BRASIL, 2018).

Peduzzi (2016) afirma que o SUS possui como característica principal a interprofissionalidade. Sendo assim, para a mesma autora (PEDUZZI, 2016), a EIP tem uma relação mútua com o sistema público de saúde no contexto brasileiro. No Brasil, entretanto, as 
PETERMANN, X. B.; MIOLO, S. B.

iniciativas para estudos de EIP nas graduações e pós-graduações ainda são incipientes e envolvem contextos isolados.

As principais iniciativas de EIP no Brasil compreendem o Programa de Reorientação da Formação do Profissional da Saúde (PRÓ-Saúde), lançados em 2005 pela assinatura da portaria interministerial do Ministério da Saúde e do Ministério da Educação (BRASIL, 2007) e o Programas de Educação pelo Trabalho para a Saúde (PET-Saúde), no âmbito dos ministérios da Saúde e da Educação, destinados a fomentar grupos de aprendizagem tutorial em áreas estratégicas para o SUS nas graduações em saúde (BRASIL, 2010). Na pós-graduação surgiram os Programas de Residência Multiprofissional em Saúde (PRMS) com o intuito de ampliar a formação fundamentada na atenção integral e interprofissional (BRASIL, 2005).

Guraya e Barr (2018), em estudo de revisão sistemática, descreveram que ocorreu um impacto positivo na intervenção educacional pelo programa de EIP em várias disciplinas da saúde, no entanto mais estudos são necessários para identificar o efeito da EIP na competência clínica dos alunos e profissionais da saúde. Complementando, Dyess et al. (2019) e Wang et al. (2019), em estudos de revisão sistemática, relataram que as evidências sugerem que a intervenção por meio da EIP teve efeitos positivos na formação de profissionais da saúde, pois impactou na melhora das atitudes em relação ao trabalho em equipe interdisciplinar, comunicação, resolução compartilhada de problemas, conhecimento e habilidades na preparação para a colaboração em equipes interdisciplinares.

Diante disso, emerge a seguinte questão de pesquisa que motivou a realização deste estudo: Qual a produção científica sobre a educação interprofissional no âmbito brasileiro no ensino superior? Dessa forma, este estudo teve por objetivo analisar a produção científica sobre educação interprofissional em saúde no Brasil no contexto do ensino superior.

Não há intenção, neste artigo, de esgotar o tema, exaurir possiblidades ou estabelecer moldes para a EIP no Brasil. Considera-se que um estudo de revisão integrativa da literatura sobre a temática poderá gerar suportes teóricos para auxiliar práticas relacionadas com a EIP no ensino superior no Brasil, o que é de extrema importância para a formação de profissionais em saúde com capacidade de atuar de forma interprofissional no cotidiano dos serviços de saúde, tendo impacto positivo sobre o cuidado prestado para a população. 
A estrutura deste artigo contempla, além desta introdução, um item que apresenta o percurso metodológico do estudo e outro que descreve os resultados e as devidas discussões. Por fim, retomam-se os objetivos do estudo, indicando algumas reflexões e conclusões com base nos resultados encontrados.

\section{Metodologia}

Trata-se de um estudo de revisão integrativa da literatura sobre o tema da EIP no ensino superior no contexto brasileiro. Esse tipo de revisão se caracteriza como a mais ampla abordagem metodológica no que se refere aos tipos de revisão de literatura; sendo assim, permite a inclusão de pesquisas experimentais e não experimentais com o objetivo de uma compreensão completa do objeto de estudo (SOUZA et al., 2010). Para isso, o processo de construção desse estudo seguiu as seis etapas descritas por Souza et al. (2010) para estudos de revisão integrativa: i) elaboração da questão de pesquisa; ii) amostragem ou busca na literatura; iii) coleta de dados; iv) análise crítica dos estudos incluídos; v) discussão dos resultados; e vi) apresentação da revisão integrativa.

A busca bibliográfica foi realizada em outubro de 2020 nas bases de dados Literatura Latino-Americana e do Caribe em Ciências da Saúde (LILACS) e Scientific Electronic Library Online (SciELO). Os termos utilizados para a busca dos artigos, de acordo com os descritores em ciências da saúde (DeCS), foram: "educação interprofissional and saúde and educação superior". Os termos poderiam estar em qualquer parte do manuscrito.

Como critérios de inclusão foram adotados: artigos que tratassem da temática da educação interprofissional no Brasil no contexto do ensino superior; disponibilidade completa on-line; idiomas português, inglês e espanhol; período de 2004 a 2020. Considerou-se o ano 2004 o marco inicial, quando foi instituída a PNEPS (BRASIL, 2004), criando possibilidades para o desenvolvimento da EIP no Brasil. Os critérios de exclusão seguidos foram: teses, dissertações, trabalhos de conclusão de curso e cartas aos editores; capítulos de livros e relatos de experiência; e estudos sobre outros assuntos que não se relacionam com o tema desta revisão.

Após a busca dos artigos nas bases de dados, de acordo com a questão de pesquisa - Qual a produção científica sobre a educação interprofissional no âmbito brasileiro no ensino 
superior? - e com os critérios de inclusão previamente descritos, procedeu-se à leitura dos títulos e resumos dos estudos e, quando necessária, a leitura do artigo completo. Após essa etapa, foram excluídos os artigos conforme os critérios de exclusão previamente definidos, bem como os artigos duplicados nas bases de dados.

Em seguida, os artigos incluídos foram lidos na íntegra, para, em seguida, iniciar-se a extração dos dados com as características de cada estudo: título, base de dados, periódico, ano de publicação, autores, objetivo do estudo, delineamento metodológico, principais resultados e nível de evidência.

Para a definição do nível de evidência dos artigos foram considerados os sete níveis de classificação, sendo eles: nível 1, revisão sistemática ou metanálise; nível 2, ensaio clínico controlado randomizado bem delineado; nível 3, ensaio clínico controlado sem randomização; nível 4, estudos de coorte ou caso-controle bem delineados; nível 5, revisão sistemática de estudos qualitativos e descritivos; nível 6, estudos descritivos ou qualitativos; e nível 7, opinião de autoridades ou especialistas (MELNYK; FINEOUT-OVERHOLT, 2011).

Os resultados foram avaliados pela técnica da análise temática proposta por Minayo (2010), buscando responder à questão da pesquisa. Esse tipo de análise tem três etapas (MINAYO, 2010): i) pré-análise: compreende a escolha dos documentos a serem analisados e a retomada das hipóteses dos objetivos iniciais da pesquisa; ii) exploração do material: consiste na categorização, sendo esse um processo de redução do texto às palavras ou expressões mais significativas; e iii) tratamento dos resultados obtidos e interpretação: são propostas inferências e realizadas interpretações, inter-relacionando-as com o quadro teórico inicialmente desenhado.

Para maior organização e clareza, inicialmente serão apresentados os resultados da busca dos estudos nas bases de dados para, em seguida, apresentar as características dos estudos incluídos e, por fim, os achados da análise temática, bem como as devidas discussões. 


\section{Resultados e discussão}

A síntese dos resultados obtidos nas etapas de busca dos artigos nas bases de dados é apresentada na Figura 1. Sendo assim, a amostra deste estudo de revisão foi composta por 16 artigos que abordam o tema da EIP no ensino superior no âmbito brasileiro.

Figura 1 - Etapas de busca dos estudos nas bases de dados.

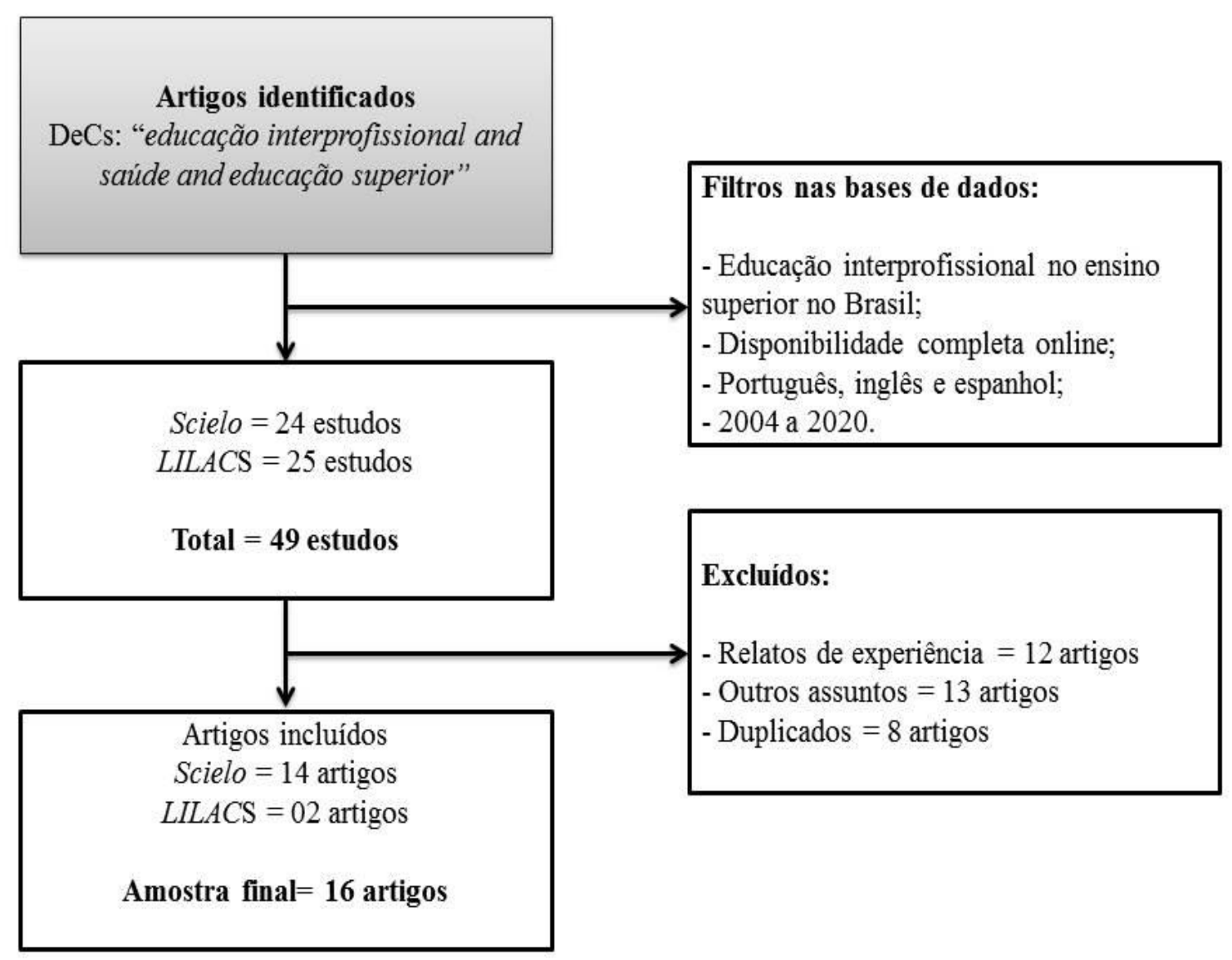

Fonte: Dados da pesquisa (2020).

Dos artigos selecionados para esta revisão integrativa, uma tabela foi desenvolvida (Tabela 1) apresentando as características segundo base de dados, autor principal, ano de 
publicação, periódico, título do estudo, método e nível de evidência (NE). Percebe-se um maior número de publicações no ano de $2018(n=6)$, na Revista Interface (Botucatu) $(n=8)$ e na SciELO $(n=14)$. Quanto ao método, prevalecem pesquisas descritivas $(n=9)$, e todos os estudos têm nível de evidência 6 , o que caracteriza as pesquisas em descritivas ou qualitativas de acordo Melnyk e Fineout-Overholt (2011).

Acredita-se que o maior número de publicações esteja concentrado no ano de 2018 devido ao plano de ação instituído pelo Ministério da Saúde (BRASIL, 2018) que fomentou a publicação científica sobre o tema, pois algumas das linhas desse plano seriam o fortalecimento e a divulgação da produção do conhecimento científico sobre EIP no Brasil. Salienta-se que a busca foi realizada a partir de 2004, entretanto os primeiros estudos publicados nas bases de dados utilizadas foram apenas depois de 2011.

Em seguida, o periódico líder em publicações sobre o tema foi a Revista Interface (Botucatu). Esse periódico, lançado em 1997 e editado pela Universidade Estadual Paulista (UNESP), caracteriza-se como uma revista científica interdisciplinar dirigida para a Educação e a Comunicação nas práticas em saúde, a formação dos profissionais da saúde e a Saúde Coletiva articulada com a Filosofia, as Artes e as Ciências Sociais e Humanas.

Ainda sobre a caracterização da produção, a base de dados que apresentou maior número de publicações foi a SciELO, cuja biblioteca eletrônica abrange uma coleção selecionada de periódicos científicos brasileiros, tendo por objetivo a preparação, o armazenamento, a disseminação e a avaliação da produção científica.

Tabela 1 - Artigos selecionados $(n=16)$.

\begin{tabular}{|c|c|c|c|c|c|c|}
\hline Base & Autor & Ano & Periódico & Título & Método & $\mathbf{N E}$ \\
\hline SciELO & $\begin{array}{l}\text { Griggio } \\
\text { et al. }\end{array}$ & 2020 & $\begin{array}{l}\text { Revista Latino- } \\
\text { Americana de } \\
\text { Enfermagem }\end{array}$ & $\begin{array}{l}\text { Analysis of an interprofissional } \\
\text { education activity in the occupational } \\
\text { health field. }\end{array}$ & Pesquisa ação. & 6 \\
\hline SciELO & $\begin{array}{l}\text { Lima et } \\
\text { al. }\end{array}$ & 2020 & $\begin{array}{l}\text { Revista Latino- } \\
\text { Americana de } \\
\text { Enfermagem }\end{array}$ & $\begin{array}{l}\text { Perception and manifestation of } \\
\text { collaborative competencies among } \\
\text { undergraduate health students. }\end{array}$ & $\begin{array}{l}\text { Pesquisa } \\
\text { intervenção. }\end{array}$ & 6 \\
\hline
\end{tabular}


Educação interprofissional em saúde no ensino superior: revisão integrativa sobre a experiência brasileira

\begin{tabular}{|c|c|c|c|c|c|c|}
\hline SciELO & $\begin{array}{l}\text { Costa et } \\
\text { al. }\end{array}$ & 2019 & $\begin{array}{l}\text { Saúde em } \\
\text { Debate }\end{array}$ & $\begin{array}{l}\text { Aspectos institucionais para a adoção } \\
\text { da Educação Interprofissional na } \\
\text { formação em enfermagem e } \\
\text { medicina. }\end{array}$ & $\begin{array}{l}\text { Estudo de caso } \\
\text { qualitativo. }\end{array}$ & 6 \\
\hline SciELO & $\begin{array}{l}\text { Magnago } \\
\text { et al. }\end{array}$ & 2019 & $\begin{array}{l}\text { Saúde em } \\
\text { Debate }\end{array}$ & $\begin{array}{l}\text { PET-Saúde/GraduaSUS na visão de } \\
\text { atores do serviço e do ensino: } \\
\text { contribuições, limites e sugestões. }\end{array}$ & $\begin{array}{l}\text { Descritivo } \\
\text { exploratório. }\end{array}$ & 6 \\
\hline SciELO & $\begin{array}{l}\text { Casanov } \\
\text { a et al. }\end{array}$ & 2018 & $\begin{array}{l}\text { Interface } \\
\text { (Botucatu) }\end{array}$ & $\begin{array}{l}\text { A Educação Interprofissional e a } \\
\text { prática compartilhada em programas } \\
\text { de residência multiprofissional em } \\
\text { Saúde }\end{array}$ & $\begin{array}{l}\text { Exploratório } \\
\text { descritivo. }\end{array}$ & 6 \\
\hline SciELO & $\begin{array}{l}\text { Santos et } \\
\text { al. }\end{array}$ & 2018 & $\begin{array}{l}\text { Interface } \\
\text { (Botucatu) }\end{array}$ & $\begin{array}{l}\text { A educação interprofissional na } \\
\text { graduação de Medicina e } \\
\text { Enfermagem em prática na atenção } \\
\text { primária à saúde: a perspectiva dos } \\
\text { estudantes }\end{array}$ & $\begin{array}{l}\text { Qualitativo } \\
\text { descritivo. }\end{array}$ & 6 \\
\hline SciELO & $\begin{array}{l}\text { Santos et } \\
\text { al. }\end{array}$ & 2018 & $\begin{array}{l}\text { Interface } \\
\text { (Botucatu) }\end{array}$ & $\begin{array}{l}\text { Docência, Pró-Saúde e } \text { PET-Saúde: } \\
\text { narrativas de um fazer } \\
\text { interprofissional. }\end{array}$ & $\begin{array}{l}\text { Qualitativo } \\
\text { descritivo. }\end{array}$ & 6 \\
\hline SciELO & $\begin{array}{l}\text { Barreto } \\
\text { et al. }\end{array}$ & 2018 & $\begin{array}{l}\text { Interface } \\
\text { (Botucatu) }\end{array}$ & $\begin{array}{l}\text { Integração de instituições de ensino } \\
\text { superior com sistemas municipais de } \\
\text { saúde à luz de uma tipologia da } \\
\text { colaboração interprofissional. }\end{array}$ & Estudo de caso. & 6 \\
\hline SciELO & Eli et al. & 2018 & $\begin{array}{l}\text { Interface } \\
\text { (Botucatu) }\end{array}$ & $\begin{array}{l}\text { Integração entre currículos na } \\
\text { educação de profissionais da Saúde: a } \\
\text { potência para } \quad \text { educação } \\
\text { interprofissional na graduação. }\end{array}$ & Estudo de caso. & 6 \\
\hline LILACS & $\begin{array}{l}\text { Paro et } \\
\text { al. }\end{array}$ & 2018 & $\begin{array}{l}\text { Interface } \\
\text { (Botucatu) }\end{array}$ & $\begin{array}{l}\text { Interprofissionalidade na graduação } \\
\text { em Saúde Coletiva: olhares a partir } \\
\text { dos cenários diversificados de } \\
\text { aprendizagem. }\end{array}$ & $\begin{array}{l}\text { Qualitativo } \\
\text { exploratório. }\end{array}$ & 6 \\
\hline SciELO & $\begin{array}{l}\text { Nuto et } \\
\text { al. }\end{array}$ & 2017 & $\begin{array}{l}\text { Rev. Bras. } \\
\text { Educ. Med. }\end{array}$ & $\begin{array}{l}\text { Avaliação da disponibilidade para } \\
\text { aprendizagem interprofissional de }\end{array}$ & $\begin{array}{l}\text { Transversal, } \\
\text { observacional, }\end{array}$ & 6 \\
\hline
\end{tabular}




\begin{tabular}{|c|c|c|c|c|c|c|}
\hline & & & & estudantes de Ciências da Saúde. & $\begin{array}{ll}\text { descritivo } & \mathrm{e} \\
\text { quantitativo. } & \end{array}$ & \\
\hline SciELO & $\begin{array}{l}\text { Rocha et } \\
\text { al. }\end{array}$ & 2016 & $\begin{array}{l}\text { Interface } \\
\text { (Botucatu) }\end{array}$ & $\begin{array}{l}\text { Colaboração interprofissional: estudo } \\
\text { de caso entre gestores, docentes e } \\
\text { profissionais de saúde da família. }\end{array}$ & $\begin{array}{l}\text { Estudo de caso, } \\
\text { qualitativo, } \\
\text { exploratório. }\end{array}$ & 6 \\
\hline LILACS & $\begin{array}{l}\text { Santos et } \\
\text { al. }\end{array}$ & 2015 & $\begin{array}{l}\text { ABCS Health } \\
\text { Sciences }\end{array}$ & $\begin{array}{l}\text { Monitoria acadêmica na formação } \\
\text { em/para a saúde: desafios e } \\
\text { possibilidades no âmbito de um } \\
\text { currículo interprofissional em saúde }\end{array}$ & $\begin{array}{l}\text { Qualitativo } \\
\text { descritivo. }\end{array}$ & 6 \\
\hline SciELO & $\begin{array}{l}\text { Costa et } \\
\text { al. }\end{array}$ & 2015 & $\begin{array}{l}\text { Interface } \\
\text { (Botucatu) }\end{array}$ & $\begin{array}{llrl}\text { Pró-Saúde e } & \text { PET-Saúde como } \\
\text { espaços } & \text { de } & \text { educação } \\
\text { interprofissional. } & \end{array}$ & $\begin{array}{l}\text { Quantitativo } \\
\text { descritivo. }\end{array}$ & 6 \\
\hline SciELO & $\begin{array}{l}\text { Souto et } \\
\text { al. }\end{array}$ & 2014 & $\begin{array}{l}\text { Psicol. Cienc. } \\
\text { Prof. }\end{array}$ & $\begin{array}{l}\text { A educação interprofissional na } \\
\text { formação em Psicologia: olhares de } \\
\text { estudantes. }\end{array}$ & $\begin{array}{l}\text { Qualitativo } \\
\text { descritivo. }\end{array}$ & 6 \\
\hline SciELO & $\begin{array}{l}\text { Aguilar } \\
\text { da Silva } \\
\text { et al. }\end{array}$ & 2011 & $\begin{array}{l}\text { Avaliação } \\
\text { (Campinas) }\end{array}$ & $\begin{array}{l}\text { Avaliação } \text { da formação } \\
\text { interprofissional no ensino superior } \\
\text { em saúde: aspectos da colaboração e } \\
\text { do trabalho em equipe. }\end{array}$ & Descritivo. & 6 \\
\hline
\end{tabular}

Legenda: NE, nível de evidência).

Fonte: dados da pesquisa (2020).

Após a descrição das principais características dos estudos incluídos, parte-se para os resultados da análise temática do capítulo denominado resultados dos estudos. Dessa etapa emergiram três categorias que caracterizam a produção científica sobre EIP em saúde no ensino superior no Brasil: "EIP como meio para a troca de saberes", "EIP como espaço de reflexão" e "EIP como elemento para o cuidado integral", descritas a seguir. As categorias, bem como os elementos que as elegeram, apresentaram-se inter-relacionadas no contexto da EIP. Nesse sentido, a Figura 2 ilustra as categorias que retratam a produção científica sobre EIP no Brasil no ensino superior. 
Figura 2 - Fatores relacionados à EIP no ensino superior no Brasil.

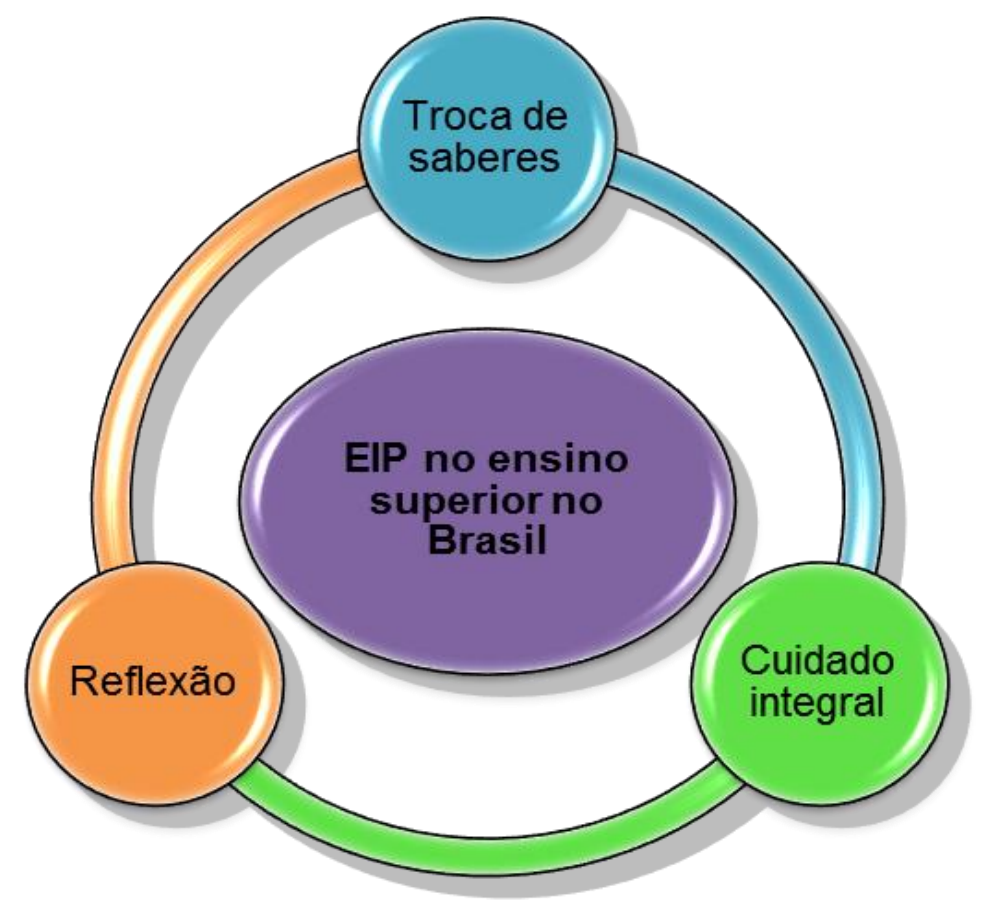

Fonte: Dados da pesquisa (2020).

A partir disso, apresentam-se as análises de cada uma das categorias identificadas pela avaliação temática, permitindo identificar os fatores que contribuíram para a eleição dessas categorias, bem como as devidas discussões.

\subsection{A EIP como meio para a troca de saberes}

Referente à EIP como meio para a troca de saberes, percebeu-se que as publicações convergiram para o compartilhamento de práticas, integração e interação profissional. Salientase que essa categoria foi identificada na maioria das publicações incluídas nesta revisão sobre o tema. Sendo assim, infere-se que a troca de saberes é um fator importante relacionado com a EIP e que deve ser considerado no processo de planejamento.

Os principais fatores associados à EIP como meio para a troca de saberes estão resumidos no Quadro 1. 
Quadro 1 - Troca de saberes.

\begin{tabular}{|l|l|}
\hline \multirow{2}{*}{ EIP como meio para a troca de saberes } & Compartilhamento de práticas \\
\cline { 2 - 2 } & Integração e interação profissional \\
\hline
\end{tabular}

Fonte: Dados da pesquisa (2020).

Corroborando os resultados deste estudo, a principal contribuição da EIP para Batista $e t$ al. (2016) também compreende a experiência com aprendizagens interativas, destacando que essas iniciativas sejam integradas nos currículos dos cursos. Ao encontro dos significados, a OMS (2010) descreve a EIP como aprendizado em conjunto entre diferentes profissões. Documento recente retrata que a EIP representa uma oportunidade para o processo de formação conjunta, com o intuito de fomentar aprendizagens compartilhadas e impactar de maneira positiva a qualidade da assistência (KHALILI; HALL; DELUCA, 2019).

Franco et al. apud Merhy et al. (2007) descrevem o imperativo de articular o conhecimento nas equipes de saúde para potencializar o aprendizado das dinâmicas complexas de relações e interações com os usuários e com a própria equipe de saúde. Reconhecem, também, a importância do uso das tecnologias leves, do desenvolvimento de conhecimentos técnicos e de novas configurações do trabalho em saúde. Hall (2005) aponta para os desafios de a EIP se associar às culturas das profissões com base no cuidado uniprofissional, pois ainda não compartilham uma mesma cultura profissional.

Estudos internacionais (KENT; KEATING, 2015; GURAYA; BARR, 2018; ZECHARIAH et al., 2019) indicam que um programa de EIP possibilita aos discentes e profissionais da saúde o aprendizado com outras disciplinas. A partir dos achados desta revisão e dos estudos nacionais e internacionais apresentados, nota-se que a troca de saberes é caracterizada como um importante instrumento da EIP. 


\subsection{A EIP como espaço para reflexão}

As publicações sobre o tema da EIP no ensino superior no Brasil retrataram-na como espaço para reflexão, e como elementos-chave dessa categoria estão o aprendizado, a construção de saberes e a qualificação profissional. Essa perspectiva pode ser visualizada no Quadro 2.

Quadro 2 - Espaço para reflexão.

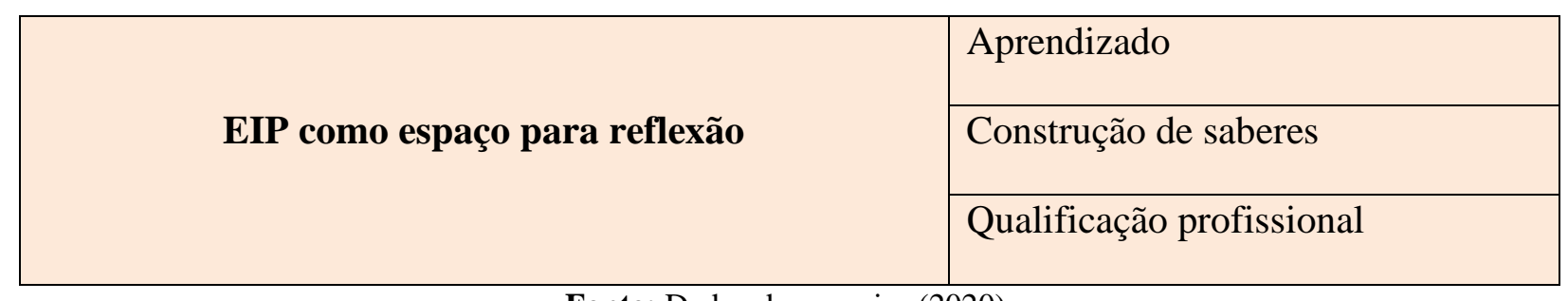

Fonte: Dados da pesquisa (2020).

Rossit et al. (2014) descrevem a EIP no contexto do processo de formação como um mecanismo para estimular que um futuro profissional seja um sujeito mais crítico e reflexivo, comprometido com a qualidade da atenção, bem como com uma visão mais sensível ao contexto socioeconômico e cultural dos indivíduos e coletividades. Nessa perspectiva, a contribuição de experiências com base na EIP favorece as discussões entre os profissionais e, dessa maneira, as decisões pautadas nas necessidades de saúde da população com olhares de diferentes núcleos profissionais.

Apesar dos diversos avanços da EIP no processo de ensino-aprendizagem nos cursos de graduação e pós-graduação em saúde, Oliveira et al. (2016) apontam que ainda existem poucos projetos de uma formação interprofissional. Acredita-se que iniciativas de EIP devem ser valorizadas no processo formativo, pois as práticas profissionais no cotidiano dos serviços, em especial no SUS, exigem atitudes e habilidades de relações interprofissionais.

Matuda et al. (2013) relatam que, no SUS, a atenção à saúde dos usuários é uma ação cada vez mais complexa, que demanda a interação dos profissionais da saúde, e que, apesar de poucos estudos empíricos sobre a efetividade da EIP, os resultados desse modelo são usados por 
gestores como uma das prioridades para a melhoria do processo de trabalho com as equipes de saúde.

Além disso, outros estudos (REEVES et al., 2016; WANG et al., 2019) corroboram esses resultados, pois comprovaram aumentos no conhecimento e nas habilidades colaborativas por meio da EIP.

\subsection{A EIP como elemento para o cuidado integral}

Os artigos analisados comprovam a EIP como elemento para o cuidado integral da população, por meio de uma atenção baseada nas necessidades do sujeito e na qualidade do cuidado em saúde (Quadro 3).

Quadro 3 - Cuidado integral.

\begin{tabular}{|l|l|}
\hline \multirow{2}{*}{ EIP como elemento para o cuidado integral } & Cuidado pautado nas necessidades do sujeito \\
\cline { 2 - 2 } & Atenção de qualidade \\
\hline
\end{tabular}

Fonte: Dados da pesquisa (2020).

Em concordância com os achados deste estudo, Forte et al. (2016) descrevem que a EIP possui como principal enfoque a integralidade, princípio doutrinário do SUS, permitindo um trabalho que envolve a prevenção e a promoção da saúde, com uma compreensão ampliada do processo de saúde-doença. Os mesmos autores apontam para a EIP como uma maneira de inventar e reinventar as formas de atuação dos profissionais com o objetivo de melhores resultados no cuidado em saúde.

Madruga et al. (2015) confirmam que ações pautadas na EIP têm maior potencial de ampliar a visão do processo de saúde-doença e, assim, da assistência integral ao sujeito. Sem dúvida, o propósito final da EIP é auxiliar na formação de interprofissionais que atuem em prol de melhores condições de saúde para a população por meio de um cuidado integral e humanizado. Matuda et al. (2013) também reconhecem a EIP como um meio para evitar a 
desumanização e os riscos da assistência à saúde, contribuindo para aumentar a qualidade do cuidado e colocando no centro as necessidades dos usuários.

O Ministério da Saúde (2019) também destaca a importante contribuição da EIP como pilar para a qualidade da atenção à saúde no Brasil para o efetivo trabalho em equipe e a promoção da integralidade em saúde. Anteriormente, a OMS já havia mencionado a contribuição da EIP para a qualificação da assistência em saúde em nível mundial e para o cuidado integral em saúde. Nota-se que importantes órgãos relacionados com o sistema público de saúde convergem para o mesmo olhar - EIP como elemento para o cuidado integral.

Reeves et al. (2017), entretanto, indicam que esse campo ainda está se desenvolvendo, havendo a necessidade de mais estudos rigorosos de métodos mistos para avaliar os efeitos da EIP na prática clínica e, também, na saúde dos usuários. Para isso sugerem estudos longitudinais, mais rigorosos e com métodos mistos.

Sendo assim, a revisão integrativa da literatura dos 16 artigos permitiu que se obtivesse um panorama da produção acadêmica nacional por meio das bases indexadoras SciELO e LILACS, de modo a direcionar ainda as lacunas existentes na produção e a consequente evolução da temática da EIP no país no período de 2004 a 2020.

\section{Conclusão}

Com base nos resultados deste artigo de revisão integrativa, que teve por objetivo analisar a produção científica sobre EIP em Saúde no Brasil no contexto do ensino superior, percebeu-se que as publicações incluídas retratam perspectivas de EIP fundamentadas na troca de saberes, em espaços de reflexão e elementos para o cuidado integral.

Os conceitos e perspectiva, aqui encontrados demonstraram que a EIP compreendeu uma proposta efetiva, com impacto positivo sobre o processo de ensino e aprendizado na educação superior e, consequentemente, sobre a qualidade da atenção prestada na assistência à saúde, em especial no contexto público. Existe, no entanto, a necessidade de pesquisas com maior nível de evidência sobre a EIP, em especial no contexto brasileiro. 
Como contribuição, um estudo de revisão integrativa apresenta a atual situação da produção científica acerca da EIP no ensino superior no Brasil no período de 2004 a 2020 nas bases SciELO e LILACS. Pode-se considerar limitação deste estudo a inclusão apenas de artigos de periódicos brasileiros, sendo interessante o mapeamento de estudos brasileiros publicados em periódicos internacionais, o que permitiria investigar um cenário mais abrangente e profundo sobre a EIP no contexto do ensino superior no Brasil.

\section{Referências}

BATISTA, N. A.; BATISTA, S. H. S. da S. Educação interprofissional na formação em saúde: tecendo redes de práticas e saberes. Interface - Comunicação, Saúde, Educação, v. 20, p. 202204, 2016.

BRASIL. Ministério da Saúde. Educação Interprofissional. Disponível em: http://www.saude.gov.br/trabalho-educacao-e qualificacao/gestao-da-educacao/qualificacaoprofissional/44937-educacao-interprofissional. Acesso em: 15 dez. 2019.

BRASIL. Ministério da Saúde. Lei no 11.129, de 30 de junho de 2005. Art. 13. Institui a Residência em Área Profissional da Saúde; Art. 14. Cria no âmbito do Ministério da Educação a Comissão Nacional de Residência Multiprofissional em Saúde. Diário Oficial da União de 01/07/2005.

BRASIL. Ministério da Saúde. Portaria Interministerial n 421, de 03 de Março de 2010. Institui o Programa de Trabalho para a Saúde (PET-Saúde) e dá outras providências.

BRASIL. Ministério da Saúde, Secretaria de Gestão do Trabalho e da Educação na Saúde. Departamento de Gestão da Educação na Saúde. Política Nacional de Educação Permanente em Saúde: o que se tem produzido para o seu fortalecimento? Brasília, DF: Ministério da Saúde; 2018.

BRASIL. Ministério da Saúde/Ministério da Educação. Programa Nacional de Reorientação da Formação Profissional em Saúde. Pró-Saúde: Objetivos, implementação e desenvolvimento potencial. Brasília: Ministério da Saúde; 2007.

CAMARA, A. M. C. S.; GROSSEMAN, S.; PINHO, D. L. M. Educação interprofissional no Programa PET-Saúde: a percepção de tutores. Interface - Comunicação, Saúde, Educação, v. 19, p. 817-829, 2015.

DYESS, A. L. et al. Impact of interprofessional education on students of the health professions: a systematic review. Journal of Educational Evaluation for Health Professions, v. 16, 2019. 
FORTE, F. D. S. et al. Educação interprofissional e o programa de educação pelo trabalho para a saúde/Rede Cegonha: potencializando mudanças na formação acadêmica. Interface Comunicação, Saúde, Educação, v. 20, p. 787-796, 2016.

FRANCO, T. B.; MERHY, E. E. Programa de Saúde da Família (PSF): contradições de um programa destinado à mudança do modelo tecnoassistencial. $\mathrm{O}$ trabalho em saúde: olhando e experienciando o SUS no cotidiano. São Paulo: Hucitec, p. 55-124, 2003.

GURAYA, S. Y.; BARR, H. The effectiveness of interprofessional education in healthcare: A systematic review and meta-analysis. The Kaohsiung journal of medical sciences, v. 34, n. 3, p. 160-165, 2018.

HALL, P. Interprofessional teamwork: Professional cultures as barriers. Journal of Interprofessional Care, v. 19, n. sup1, p. 188-196, 2005.

KENT, F.; KEATING, J. L. Interprofessional education in primary health care for entry level students: A systematic literature review. Nurse Education Today, v. 35, n. 12, p. 1221-1231, 2015 .

KHALILI, H., HALL, J., DELUCA, S. Orientação para a educação interprofissional global e pesquisa sobre a prática colaborativa: Documento de trabalho. 2019. Publicação conjunta do Interprofessional Research Global e da Interprofessional Global. Disponível em: www. research.interprofessional.global. Acesso em: 2 out. 2019.

MADRUGA, L. M. de S. et al. O PET-Saúde da Família e a formação de profissionais da saúde: a percepção de estudantes. Interface - Comunicação, Saúde, Educação, v. 19, p. 805-816, 2015.

MATUDA, C. G.; AGUIAR, D. M. de L.; FRAZÃO, P. Cooperação interprofissional e a Reforma Sanitária no Brasil: implicações para o modelo de atenção à saúde. Saúde e Sociedade, v. 22, p. 173-186, 2013.

MELNYK, B. M.; FINEOUT-OVERHOLT, E. (ed.). Evidence-based practice in nursing \& healthcare: a guide to best practice. Lippincott Williams \& Wilkins, 2011.

MINAYO, M. C. de S. Introdução à metodologia das ciências sociais. O desafio do conhecimento: pesquisa qualitativa em saúde. 12. ed. São Paulo (SP): Hucitec. 2010.

OLIVEIRA, M. de L. S. de; SILVA, F. C. S. Saúde Coletiva: o olhar do egresso sobre a formação. Revista Saúde e Desenvolvimento, v. 7, n. 4, p. 78-93, 2016.

OLSON, R.; BIALOCERKOWSKI, A. Interprofessional education in allied health: a systematic review. Medical Education, v. 48, n. 3, p. 236-246, 2014.

ORGANIZAÇÃO MUNDIAL DA SAÚDE. Marco para ação em educação interprofissional e prática colaborativa. 2010 . 
PAN AMERICAN HEALTH ORGANIZATION. Interprofessional Education in Health Care: Improving Human Resource Capacity to Achieve Universal Health. Report of the Meeting. Bogota, Colombia, 7-9 Dec 2016. Washington, DC: PAHO; 2017.

PEDUZZI, M. O SUS é interprofissional. Interface - Comunicação, Saúde, Educação, v. 20, p. 199-201, 2016.

REEVES, S. et al. A BEME systematic review of the effects of interprofessional education: BEME Guide No. 39. Medical Teacher, v. 38, n. 7, p. 656-668, 2016.

REEVES, S. et al. Interprofessional collaboration to improve professional practice and healthcare outcomes. Cochrane Database of Systematic Reviews, n. 6, 2017.

REEVES, S. Porque precisamos da educação interprofissional para um cuidado efetivo e seguro. Interface - Comunicação, Saúde, Educação, v. 20, n. 56, 2016.

ROSSIT, R.; BATISTA, S. H.; BATISTA, N. A. Training for integrality in Health Care Careers: Potential for an Interprofessional Project. Revista Internacional de Humanidades Médicas, v. 3, n. $1,2014$.

Sobre a Revista Interface. Informações básicas. Disponível em: http://www.scielo.br/revistas/icse/paboutj.htm. Acesso em: 15 dez. 2019.

Sobre a SciELO. SciELO. Disponível em: http://www.scielo.br/scielo.php?script=sci_home\&lng=pt\&nrm=iso\#about. Acesso em: 15 dez. 2019.

SOUZA, M. T. de; SILVA, M. D. da; CARVALHO, R. de. Integrative review: what is it? How to do it? Einstein, São Paulo, v. 8, n. 1, p. 102-106, 2010.

WANG, Z. et al. A Systematic Meta-Analysis of the Effect of Interprofessional Education on Health Professions Students' Attitudes. Journal of Dental Education, v. 83, n. 12, p. 1361$1369,2019$.

ZECHARIAH, S. et al. Interprofessional Education and Collaboration in Healthcare: An Exploratory Study of the Perspectives of Medical Students in the United States. Healthcare. Multidisciplinary Digital Publishing Institute, v. 7, n. 4, p. 117, 2017.

Recebido em: 19/12/2019

Revisado em: 04/11/2020

Aprovado em: 10/11/2020

Publicado em: 15/03/2021 